\title{
Quantum gravity effects on statistics and compact star configurations
}

\author{
Peng Wang1, Haitang Yang 2 and Xiuming Zhang 3 \\ Department of Applied Physics, \\ University of Electronic Science and Technology of China, \\ Chengdu, 610054, People's Republic of China
}

\begin{abstract}
The thermodynamics of classical and quantum ideal gases based on the Generalized uncertainty principle (GUP) are investigated. At low temperatures, we calculate corrections to the energy and entropy. The equations of state receive small modifications. We study a system comprised of a zero temperature ultra-relativistic Fermi gas. It turns out that at low Fermi energy $\varepsilon_{F}$, the degenerate pressure and energy are lifted. The Chandrasekhar limit receives a small positive correction. We discuss the applications on configurations of compact stars. As $\varepsilon_{F}$ increases, the radius, total number of fermions and mass first reach their nonvanishing minima and then diverge. Beyond a critical Fermi energy, the radius of a compact star becomes smaller than the Schwarzschild one. The stability of the configurations is also addressed. We find that beyond another critical value of the Fermi energy, the configurations are stable. At large radius, the increment of the degenerate pressure is accelerated at a rate proportional to the radius.
\end{abstract}

\footnotetext{
${ }^{1}$ E-mail: pengw@uestc.edu.cn

${ }^{2}$ E-mail: hyanga@uestc.edu.cn

${ }^{3}$ E-mail: zhangxm@uestc.edu.cn
} 


\section{Contents}

1 Introduction $\quad 2$

2 Ideal gases at finite temperatures 4

2.1 classical statistics . . . . . . . . . . . . . . . . . . . . . 4

2.2 The photon gas ................................

3 Ground state properties of ultra-relativistic Fermi gases 7

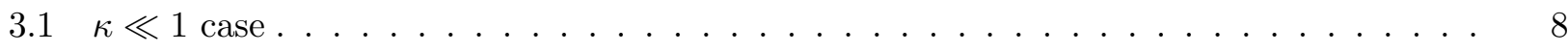

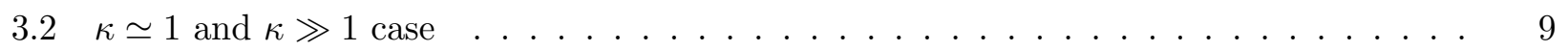

4 Ultra-compact stars at zero temperature $\quad 10$

4.1 White dwarfs with $M=2 N m_{p} \ldots \ldots \ldots \ldots \ldots \ldots$

4.2 Configurations with $M=U_{0} / c^{2} \ldots \ldots \ldots \ldots \ldots \ldots \ldots$

5 Summary and Discussion $\quad 14$

\section{Introduction}

It has been suggested that gravity itself leads to an effective cutoff in the ultraviolet, i.e., a minimal observable length [1, 2, 3, 4, 5, 6]. Some realizations of the minimal length from various scenarios are proposed. One of the most important models is the generalized uncertainty principle (GUP), derived from the modified fundamental commutation relation [7, 8, 9, 10, 11, 12, 13.

$$
[x, p]=i \hbar\left(1+\beta p^{2}\right),
$$

where $\beta=\beta_{0} \ell_{p}^{2} / \hbar^{2}=\beta_{0} / c^{2} M_{p}^{2}$ with the Planck mass $M_{p}=\sqrt{\hbar c / G}$ and the Planck length $\ell_{p}=$

$\sqrt{G \hbar / c^{3}}$. $\beta_{0}$ is a dimensionless parameter. With this generalization, one can easily derive the generalized uncertainty principle (GUP)

$$
\Delta x \Delta p \geq \frac{\hbar}{2}\left[1+\beta(\Delta p)^{2}\right] .
$$

This in turn gives the minimum measurable length

$$
\Delta x \geq \Delta_{\min }=\hbar \sqrt{\beta}=\sqrt{\beta_{0}} \ell_{p} .
$$

Eqn. (11) is the simplest model where only the minimal uncertainty in position is taken into account while the momentum can be infinite. In this case, the quantum-mechanical structure underlying the 
GUP has been studied in full detail [9]. The planar waves in momentum space are generalized by the maximal localization states and directly lead to the modified de Broglie relation

$$
\hbar k=\frac{\tan ^{-1}(\sqrt{\beta} p)}{\sqrt{\beta}} .
$$

In the limit $\beta \rightarrow 0$, one can obtain the usual de Broglie relation. For $\beta \neq 0$, there is a nonzero minimal wavelength

$$
\lambda_{0}=4 \hbar \sqrt{\beta} .
$$

Many implications and applications of non-zero minimal length have been discussed in literature [9, 14, 15]. A more detailed list of references refers to [16]. In [17], based on the precision measurement of Lamb shift, an upper bound of $\beta_{0}$ is given by $\beta_{0}<10^{36}$. A relatively rough but stronger restriction is estimated in [18]. Furthermore, in [20], the parameter $\beta_{0}$ is conjectured to vary with energy scales. In our analysis, $\beta_{0}<10^{36}$ is adopted.

In this paper, we discuss the thermodynamic properties of ideal gases based on GUP. The statistical physics of ideal gases has been studied by many authors [15, 16, 19, 21, 22]. It turns out that the thermodynamic quantities at finite temperatures are shifted by $\beta k_{\mathrm{B}} T$ for non-relativistic system or $\beta\left(k_{\mathrm{B}} T\right)^{2}$ for ultra-relativistic and photon ones. Accordingly, there exist modifications on the equations of state.

It is well known that the cold relativistic Fermi gas has important applications in astrophysics. Especially in 1930s, Chandrasekhar found that white dwarfs are very well described in the framework of a highly degenerate ideal electron gas. Therefore, it is of interest to investigate the ultra-relativistic ideal Fermi gas at zero temperature. It proves that both the energy and pressure of the system receive quantum gravity corrections as the Fermi energy is low. Since a full theory of quantum gravity is absent, it is a good try to take GUP model as a starting point and apply it to the case of high Fermi energy. We find that both the number density and the energy density approach finite values, whereas the degenerate pressure blows up.

We are going to address two kinds of compact star configurations. One is white dwarf alike where the major contribution of the mass is not from the Fermi gas under discussion but from the cold nuclei. Application of our arguments to this type of compact stars causes small corrections to the Chandrasekhar limit. The mass of another configuration is expressed as $M=U_{0} / c^{2}$, where the compact star is completely constituted by an ideal Fermi gas. Our calculation shows that the radius, total number and mass all have minima at Fermi energies around $E_{\mathrm{H}}=M_{p} c^{2} / \sqrt{\beta}_{0}$. The minimum radius is proportional to $\sqrt{\beta}_{0} \Delta_{\text {min }}$, different from the intuitively expected value $\Delta_{\text {min. }}$. The radius becomes smaller than the Schwarzschild one beyond a critical value of the Fermi energy, though eventually approaches infinity. This scenario provides a possible interpretation of our universe as a giant black hole. We further find that the degenerate pressure increases at a rate proportional to the radius asymptotically. A question therefore naturally arises: Is the accelerating expansion of our universe accounted by Fermi pressure?

We consider an isolated macroscopic body, consisting of $N$ non-interacting particles. In condensed matter physics, the background of particles' motion is flat. However, as we know from (1) and 
(3)), the generalized uncertainty principle realizes that the particles move in quantized gravitational background. It is then natural to consider the nearly independent particle systems. The state density has been derived in several ways [15, 16, 19]. In [19], with the help of Liouville theorem, the density of states is given

$$
\frac{V d p_{1} d p_{2} \cdots d p_{d}}{h^{d}\left(1+\beta p^{2}\right)^{d}}
$$

In $d$-dimensional spherical coordinate systems, the state density in momentum space is

$$
D(p) d p=\frac{V A\left(S^{d-1}\right) p^{d-1} d p}{h^{d}\left(1+\beta p^{2}\right)^{d}}, \quad p \in(0,+\infty)
$$

where $A\left(S^{d-1}\right)=2 \pi^{d / 2} / \Gamma\left(\frac{d}{2}\right)$, with $\Gamma\left(\frac{d}{2}\right)$ being the Gamma function. If the particle's spin is 1 or $1 / 2$, the two equations above should be multiplied by 2 .

Let's review the organization of this paper. In section 2 we discuss the finite temperature classical non-relativistic, ultra-relativistic and photon gases respectively. In section 3, we explore the properties of an ultra-relativistic Fermi gas at zero temperature. Section 4 is devoted to the discussion of compact star configurations. In section 5, we offer a summary and discussion.

\section{Ideal gases at finite temperatures}

The ideal gas model plays a major role in the studies of statistics. When the electromagnetic interactions between particles can be neglected, the ideal gas is a good approximation for a real system. In this section, we discuss low temperature classical non-relativistic, ultra-relativistic systems and a photon gas respectively.

\section{1 classical statistics}

For a non-relativistic system, the dispersion relation is $\varepsilon=p^{2} / 2 m$. From (7), in $d$-dimensional space, the partition function is

$$
\begin{aligned}
Z & =\frac{V A\left(S^{d-1}\right)}{h^{d}} \int_{0}^{\infty} \frac{p^{d-1} e^{-\frac{\beta_{\mathrm{B}}}{2 m} p^{2}} d p}{\left(1+\beta p^{2}\right)^{d}} \\
& =\frac{V A\left(S^{d-1}\right)}{h^{d}}\left(2 m k_{\mathrm{B}} T\right)^{\frac{d}{2}} I(\gamma, d),
\end{aligned}
$$

where $\gamma \equiv 2 m \beta / \beta_{\mathrm{B}}=2 m \beta k_{\mathrm{B}} T$ is a dimensionless parameter in the natural unit system and $\beta_{B}=$ $1 / k_{B} T$ with $k_{\mathrm{B}}$ being the Boltzmann's constant. $I(\gamma, d)$ is defined as

$$
\begin{aligned}
I(\gamma, d) & =\frac{2^{-d} \sqrt{\pi} \gamma^{-d / 2} \Gamma\left[\frac{d}{2}\right] H\left[\frac{d}{2}, 1-\frac{d}{2}, \frac{1}{\gamma}\right]}{\Gamma\left[\frac{d+1}{2}\right]}+\frac{1}{2} \gamma^{-d} \Gamma\left[-\frac{d}{2}\right] H\left[d, 1+\frac{d}{2}, \frac{1}{\gamma}\right] \\
& \approx \frac{1}{2} \Gamma\left[\frac{d}{2}\right]\left(1-\frac{d^{2}}{2} \gamma\right)+O\left(\gamma^{2}\right),
\end{aligned}
$$


where $H[,$,$] is the Kummer confluent hypergeometric function. It is straightforward to derive the$ equation of state of the ideal gas $P V=N k_{\mathrm{B}} T$ from eqn. (8). For simplicity, we consider $d=3$,

$$
I(\gamma \ll 1,3)=\frac{\sqrt{\pi}}{4}\left[1-\frac{9}{2} \gamma\right]+O\left(\gamma^{2}\right) .
$$

In [17, an upper limit of $\beta_{0}$ is estimated as $\beta_{0}<10^{36}$ or $\beta<10^{34}\left(\frac{\mathrm{s}}{\mathrm{kg} \cdot \mathrm{m}}\right)^{2}$. If choosing $m$ to be the electron mass, $m=10^{-30} \mathrm{~kg}$, one has

$$
\frac{\gamma}{T}<10^{-19} \mathrm{~K}^{-1}
$$

In the case of low temperature, $0<T \ll 10^{19} \mathrm{~K}$, i.e., $\gamma \ll 1$, from eqn. (8) and eqn. (10), we have

$$
Z \approx \frac{4 \pi V}{h^{3}}\left(2 m k_{\mathrm{B}} T\right)^{3 / 2} \frac{\sqrt{\pi}}{4}\left[1-\frac{9}{2} \gamma\right]
$$

When $\beta=0$, one recovers the canonical energy $U_{0}=\frac{3}{2} N k_{\mathrm{B}} T$ and the entropy

$$
S_{0}=N k_{\mathrm{B}}\left[\frac{5}{2}+\frac{3}{2} \ln \frac{2 \pi m k_{\mathrm{B}} T}{h^{2}}+\ln \frac{V}{N}\right] .
$$

We are concerned with $\beta \neq 0$ where the quantum gravity effects are introduced. Combined with $0<T \ll 10^{19} \mathrm{~K}$, the energy gains a small correction

$$
U=\frac{3}{2} N k_{\mathrm{B}} T+\delta U, \quad \delta U=-\frac{9}{2} N k_{\mathrm{B}} T 2 \beta m k_{\mathrm{B}} T .
$$

Notice that the energy has nothing to do with the volume. This is entirely consistent with the case $\beta=0$. The entropy is also modified,

$$
\begin{aligned}
S & =S_{0}+\delta S \\
\delta S & =\frac{\delta U}{T}+N k_{\mathrm{B}} \ln \left[1-\frac{9}{2} \gamma\right]=-18 N k_{\mathrm{B}}^{2} m T \beta .
\end{aligned}
$$

For a nonrelativistic system, one can calculate the Maxwellian distribution in $d>1$ at low temperatures. Using (7), the velocity probability distribution for the absolute magnitude in $d$-dimensional space takes the form

$$
d \omega_{v} \sim \frac{e^{-m v^{2} / 2 k_{\mathrm{B}} T}}{\left(1+\beta m^{2} v^{2}\right)^{d}} v^{d-1} d v .
$$

The most probable distribution of the velocity is determined by the extremum,

$$
v_{\mathrm{m}}^{2}=\frac{\sqrt{[(d+1) \gamma+2]^{2}+8(d-1) \gamma}-[(d+1) \gamma+2]}{4 \gamma} \approx \frac{(d-1) k_{\mathrm{B}} T}{m}-2 d \beta m k_{\mathrm{B}} T .
$$

Comparing with the classical statistics, there is a negative shift proportional to $\beta k_{\mathrm{B}} T$.

When the particle's rest mass is very small or the temperature is high enough, the condition $k_{\mathrm{B}} T \gg m c^{2}$ is well satisfied. It is natural to study the ultra-relativistic ideal gas with the dispersion 
$\varepsilon=c p$. Then from (7), we have

$$
\begin{aligned}
Z & =\frac{V A\left(S^{d-1}\right)}{h^{d}} \int_{0}^{\infty} \frac{p^{d-1} e^{-\beta_{\mathrm{B}} c p} d p}{\left(1+\beta p^{2}\right)^{d}} \\
& =\frac{V A\left(S^{d-1}\right)}{h^{d}}\left(\frac{k_{\mathrm{B}} T}{c}\right)^{d} \int_{0}^{\infty} \frac{x^{d-1} e^{-x} d x}{\left(1+q x^{2}\right)^{d}} \\
& \equiv \frac{V A\left(S^{d-1}\right)}{h^{d}}\left(\frac{k_{\mathrm{B}} T}{c}\right)^{d} \tilde{I}(q, d),
\end{aligned}
$$

where $q \equiv \beta\left(k_{\mathrm{B}} T\right)^{2} / c^{2}$ is a dimensionless parameter. For generic dimensions, $\tilde{I}(q, d)$ is expressed as complicated generalized hypergeometric functions. Therefore, we still focus our attention on $d=3$,

$$
\tilde{I}(q, 3)=\frac{M\left[\left\{\left\{-\frac{1}{2}\right\},\{\}\right\},\left\{\left\{0, \frac{1}{2}, \frac{3}{2}\right\},\{\}\right\}, \frac{1}{4 q}\right]}{4 \sqrt{\pi} q^{3 / 2}},
$$

where $M[$,$] is the Meijer \mathrm{G}$ function. For $q \ll 1$, we find

$$
\tilde{I}(q \ll 1,3)=2-72 q+O\left(q^{2}\right) .
$$

One can see that $q=1$ defines a temperature $k_{\mathrm{B}} T_{q}=\frac{c}{\sqrt{\beta}}$. It is believed that the minimum length is about the length of a string: $\Delta_{\min }=\hbar \sqrt{\beta} \simeq \ell_{s}$. Therefore,

$$
k_{\mathrm{B}} T_{q} \simeq \frac{c \hbar}{\ell_{s}}=4 \pi k_{\mathrm{B}} T_{\mathrm{H}},
$$

where $T_{\mathrm{H}}$ is the Hagedorn temperature of relativistic strings [23, 24]. On the other hand, the Hagedorn temperature is estimated to be $T_{\mathrm{H}} \simeq 10^{30} \mathrm{~K}$ by the traditional grand-unified string models. Thus, a lower bound of $\beta_{0}$ is imposed by $T_{\mathrm{H}}$ :

$$
\beta_{0}>10^{4} .
$$

From now on, we name $k_{\mathrm{B}} T_{q}=\frac{c}{\sqrt{\beta}}=M_{p} c^{2} / \sqrt{\beta_{0}}$ as Hagedorn energy or Hagedorn temperature, the scale where the quantum gravity effects become important. When the temperature is far below the Hagedorn temperature $q \ll 1$, for $d=3$, similar to (13) and (14), the energy and entropy acquire small corrections due to the quantum gravity effects,

$$
\begin{gathered}
U=3 N k_{\mathrm{B}} T\left[1-24 \frac{\beta}{c^{2}}\left(k_{\mathrm{B}} T\right)^{2}\right], \\
S=3 N k_{\mathrm{B}}+N k_{\mathrm{B}} \ln \left[\frac{8 \pi V}{h^{3}}\left(\frac{k_{\mathrm{B}} T}{c}\right)^{3}\right]-k_{\mathrm{B}} \ln N !-108 N k_{\mathrm{B}} \frac{\beta}{c^{2}}\left(k_{\mathrm{B}} T\right)^{2} .
\end{gathered}
$$

In (8) and (15), the integrands contain exponential functions. Given $\gamma \ll 1$ or $q \ll 1$, the integrals are dominated by the neighborhoods of the maxima of the numerators. These maxima are the order of unity. Therefore, one can expand the denominators of the integrands before performing integrations. Employing this method, the integral (15) takes the form

$$
Z \approx \frac{V A\left(S^{d-1}\right)}{h^{d}}\left(\frac{k_{\mathrm{B}} T}{c}\right)^{d}[\Gamma(d)-d \Gamma(d+2) q],
$$

which precisely agrees with eqn. (17) for $d=3$. The non-relativistic one (9) is also reproduced by this method. We are going to apply this method in the following discussion of the photon gas. 


\section{$2.2 \quad$ The photon gas}

In $d$-dimensional space, given $q=\beta\left(k_{B} T\right)^{2} / c^{2} \ll 1$, the grand partition function of the photon gas is

$$
\begin{aligned}
\ln \Xi & =-2 \int_{0}^{\infty} \frac{V A\left(S^{d-1}\right) \varepsilon^{d-1}}{(h c)^{d}\left(1+\frac{\beta}{c^{2}} \varepsilon^{2}\right)^{d}} \ln \left(1-e^{-\beta_{\mathrm{B}} \varepsilon}\right) d \varepsilon \\
& \approx 2 \frac{V A\left(S^{d-1}\right)}{(h c)^{d} \beta_{\mathrm{B}}^{d}}\left[\frac{1}{d} \zeta(d+1) \Gamma(d+1)-\frac{q d}{(d+2)} \zeta(d+3) \Gamma(d+3)\right],
\end{aligned}
$$

where $\zeta(d)$ is the Riemann Zeta function. The energy is

$$
U=\frac{2 V A\left(S^{d-1}\right)}{(h c)^{d}}\left(k_{\mathrm{B}} T\right)^{d+1} \zeta(d+1) \Gamma(d+1) \cdot\left[1-d q \frac{\zeta(d+3) \Gamma(d+3)}{\zeta(d+1) \Gamma(d+1)}\right] \equiv U_{0}+\delta U .
$$

The entropy reads

$$
S=k_{\mathrm{B}} \ln \Xi+\frac{U}{T}=S_{0}+\delta S
$$

with

$$
\delta S=-q k_{\mathrm{B}} \frac{2 V A\left(S^{d-1}\right)}{(h c)^{d}} \frac{d(d+3)}{d+2} \zeta(d+3) \Gamma(d+3)\left(k_{\mathrm{B}} T\right)^{d} .
$$

From (13), (14), (18), (19), (22) and (23), one can check that

$$
\frac{\partial S}{\partial U}=\frac{\partial S_{0}}{\partial U_{0}}+\frac{\partial \delta S}{\partial U_{0}}-\frac{\partial \delta U}{\partial U_{0}} \frac{\partial S_{0}}{\partial U_{0}}=\frac{\partial S_{0}}{\partial U_{0}}
$$

In thermodynamics, there are only three independent basic thermodynamic quantities, the temperature

$T$, inner energy $U$ and entropy $S$. After introducing GUP, $S$ and $U$ are altered, while eqn. (24) shows that the temperature does not change.

\section{Ground state properties of ultra-relativistic Fermi gases}

From the previous discussion, one can see that the deformed parameter $\beta$ and the temperature $T$ are independent. What really matters is $\beta T$ or $\beta T^{2}$ respectively. In this section, we study the ground state properties of a Fermi gas composed of $N$ ultra-relativistic electrons. For non-interacting and ultra-relativistic particles, $\varepsilon=c p$. For simplicity we only consider the space dimension $d=3$. When the temperature is higher than the particle's rest mass (in the natural units), particle pairs could be produced. In this case the grand canonical distribution oughts to be used. However, at zero or low temperatures, the vacuum effect of fermions can be neglected. Therefore, the total particle number is conserved. At zero temperature, from (7), the Fermi energy $\varepsilon_{F}$ is given by

$$
\begin{aligned}
N & =\frac{8 \pi V}{h^{3}} \int_{0}^{p_{F}} \frac{p^{2} d p}{\left(1+\beta p^{2}\right)^{3}}=\frac{8 \pi V}{(h c)^{3}} \int_{0}^{\varepsilon_{F}} \frac{\varepsilon^{2} d \varepsilon}{\left(1+\frac{\beta}{c^{2}} \varepsilon^{2}\right)^{3}} \\
& =\frac{8 \pi V}{(h c)^{3}}\left(\frac{c^{2}}{\beta}\right)^{3 / 2} \frac{1}{8}\left[\frac{\kappa\left(\kappa^{2}-1\right)}{\left(1+\kappa^{2}\right)^{2}}+\tan ^{-1}(\kappa)\right] \\
& \equiv \frac{8 \pi V}{(h c)^{3}} E_{\mathrm{H}}^{3} f(\kappa),
\end{aligned}
$$


where we defined $\kappa=\varepsilon_{F} \sqrt{\frac{\beta}{c^{2}}}=\varepsilon_{F} / E_{\mathrm{H}}$ with $E_{\mathrm{H}}$ being the Hagedorn energy introduced in last section and

$$
f(\kappa)=\frac{1}{8}\left[\frac{\kappa\left(\kappa^{2}-1\right)}{\left(1+\kappa^{2}\right)^{2}}+\tan ^{-1}(\kappa)\right] .
$$

Then the average distance between particles is

$$
\bar{d} \equiv\left(\frac{V}{N}\right)^{1 / 3}=\frac{h c}{(8 \pi)^{1 / 3}}\left(\frac{\beta}{c^{2}}\right)^{1 / 2} f(\kappa)^{-\frac{1}{3}}=\pi^{\frac{2}{3}} \Delta_{\min } f(\kappa)^{-\frac{1}{3}} .
$$

The ground-state energy of the system is

$$
\begin{aligned}
U_{0} & =\frac{8 \pi V}{(h c)^{3}} \int_{0}^{\varepsilon_{F}} \frac{\varepsilon^{3} d \varepsilon}{\left(1+\frac{\beta}{c^{2}} \varepsilon^{2}\right)^{3}} \\
& =\frac{8 \pi V}{(h c)^{3}} \frac{1}{4} \varepsilon_{F}^{4} \frac{1}{\left(1+\kappa^{2}\right)^{2}} \\
& =\frac{8 \pi V}{(h c)^{3}} E_{\mathrm{H}}^{4} \frac{1}{4} \frac{\kappa^{4}}{\left(1+\kappa^{2}\right)^{2}} .
\end{aligned}
$$

The pressure of the system is given by

$$
P=\frac{8 \pi}{(h c)^{3}} k_{\mathrm{B}} T \int_{0}^{\infty} \frac{\varepsilon^{2} d \varepsilon}{\left(1+\frac{\beta}{c^{2}} \varepsilon^{2}\right)^{3}} \ln \left(1+e^{-\frac{(\varepsilon-\mu)}{k_{\mathrm{B}} T}}\right)
$$

At zero temperature, the pressure becomes

$$
P_{0}=\frac{N}{V} \varepsilon_{F}-\frac{U_{0}}{V}
$$

Plugging in (25) and (29), one has

$$
P_{0}=\frac{8 \pi}{(h c)^{3}} E_{\mathrm{H}}^{4} g(\kappa), \quad g(\kappa) \equiv \kappa f(\kappa)-\frac{1}{4} \frac{\kappa^{4}}{\left(1+\kappa^{2}\right)^{2}} .
$$

It is easy to see that $P_{0}$ is a monotonically increasing function of $\kappa$ in the region $\kappa>0$.

The equation of state plays an indispensable role in exploring the configuration of stars and cosmology. Unfortunately, an explicit expression of the pressure in terms of $\beta$ and $N / V$ is not reachable with eqn. (25) and eqn. (32). Therefore, we address the extremal situations in the following discussions.

\section{$3.1 \kappa \ll 1$ case}

In this situation,

$$
f(\kappa)=\frac{\kappa^{3}}{3}-\frac{3}{5} \kappa^{5}+O\left(\kappa^{7}\right) .
$$

This equation enables us to solve $\kappa$ in terms of $N / V$ and $\beta$ from eqn. (25):

$$
\kappa=\left(3 \pi^{2}\right)^{1 / 3} \delta\left(1+\frac{3}{5}\left(3 \pi^{2}\right)^{2 / 3} \delta^{2}\right)+O\left(\delta^{5}\right),
$$


where we have set $\delta \equiv \Delta_{\min } / \bar{d}=(N / V)^{1 / 3} \hbar \sqrt{\beta}$. It is easy to see that $\delta \ll 1$ in this case from (27). The Fermi energy is far below the Hagedorn energy,

$$
\varepsilon_{F} \ll E_{\mathrm{H}}=\frac{c^{2}}{\sqrt{\beta_{0}}} M_{p} .
$$

With equations (29) and (32), one obtains

$$
\begin{gathered}
U_{0} / V=\frac{8 \pi}{(h c)^{3}}\left(\frac{N}{8 \pi V}\right)^{\frac{4}{3}}(h c)^{4} \cdot \frac{1}{4} \frac{\kappa^{4}}{\left(1+\kappa^{2}\right)^{2}} f(\kappa)^{-\frac{4}{3}} \\
\approx \frac{3^{4 / 3}}{4} \frac{h c}{(8 \pi)^{1 / 3}}\left(\frac{N}{V}\right)^{4 / 3}\left[1+\frac{2}{5} \kappa^{2}\right] . \\
P_{0}=\frac{8 \pi}{(h c)^{3}}\left(\frac{N}{8 \pi V}\right)^{\frac{4}{3}}(h c)^{4} \cdot f(\kappa)^{-\frac{4}{3}}\left[\kappa f(\kappa)-\frac{1}{4} \frac{\kappa^{4}}{\left(1+\kappa^{2}\right)^{2}}\right] . \\
\approx \frac{3^{1 / 3}}{4} \frac{h c}{(8 \pi)^{1 / 3}}\left(\frac{N}{V}\right)^{4 / 3}\left[1+\frac{6}{5} \kappa^{2}\right] .
\end{gathered}
$$

Replacing $\kappa$ by $\delta$, the energy and pressure both receive corrections:

$$
\begin{aligned}
& U_{0} / V \approx \frac{3^{4 / 3}}{4} \frac{h c}{(8 \pi)^{1 / 3}}\left(\frac{N}{V}\right)^{4 / 3}\left[1+\frac{2}{5}\left(3 \pi^{2}\right)^{2 / 3} \delta^{2}\right], \\
& P_{0} \approx \frac{3^{1 / 3}}{4} \frac{h c}{(8 \pi)^{1 / 3}}\left(\frac{N}{V}\right)^{4 / 3}\left[1+\frac{6}{5}\left(3 \pi^{2}\right)^{2 / 3} \delta^{2}\right] \approx \frac{1}{3}\left(\frac{U_{0}}{V}\right)\left[1+\frac{4}{5}\left(3 \pi^{2}\right)^{2 / 3} \delta^{2}\right] .
\end{aligned}
$$

Thus corrections occur in the equation of state and this could lead to some cosmological consequences.

\section{$3.2 \kappa \simeq 1$ and $\kappa \gg 1$ case}

Since the full theory is absent, we take eqn. (11) as the starting point to gain some features of the quantum theory of gravity. Therefore, there is effectively no upper limit on $p^{2}$. The $\kappa \ll 1$ case corresponds to perturbative region while $\kappa \geq 1$ is equivalent to $\beta p^{2} \geq 1$.

Note that in (26),$f(\kappa)$ is a monotonically increasing and bounded function of $\kappa$ in the region $\kappa \geq 0$. So is $N / V$ with respect to $\varepsilon_{F}$. At the Hagedorn energy, $\kappa=1$ gives $f(\kappa)=\pi / 32 . \kappa \gg 1$ leads to an upper limit of $f(\kappa)$, which is $\pi / 16$. Then at zero temperature,

$$
\begin{aligned}
& \frac{N}{V}(\kappa=1)=\frac{8 \pi}{(h c)^{3}} E_{\mathrm{H}}^{3} \frac{\pi}{32}=\frac{1}{32 \pi} \frac{1}{\Delta_{\text {min }}^{3}}, \\
& \frac{N}{V}(\kappa \gg 1)=\frac{8 \pi}{(h c)^{3}} E_{\mathrm{H}}^{3} \frac{\pi}{16}=\frac{1}{16 \pi} \frac{1}{\Delta_{\text {min }}^{3}} .
\end{aligned}
$$

From (29), the energies of the ideal Fermi gases are

$$
\begin{aligned}
U_{0}(\kappa=1) & =\frac{8 \pi V}{(h c)^{3}} \frac{E_{\mathrm{H}}^{4}}{16}, \\
U_{0}(\kappa \gg 1) & =\frac{8 \pi V}{(h c)^{3}} \frac{E_{\mathrm{H}}^{4}}{4} .
\end{aligned}
$$


With (32), the pressures are

$$
\begin{aligned}
P_{0}(\kappa=1) & =\frac{\pi-2}{2} \frac{1}{V} U_{0}(\kappa=1), \\
P_{0}(\kappa \gg 1) & =\left(\frac{\pi}{4} \kappa-1\right) \frac{1}{V} U_{0}(\kappa \gg 1) .
\end{aligned}
$$

From (41) and (43), we can see that the particle number density and the energy density are bounded from above by the minimal length. However, from (45), with the increasing of the Fermi energy, the Fermi degenerate pressure blows up. This divergence indicates that more and more energy is needed to push a particle into the system. Furthermore, the equations of state are also altered.

\section{Ultra-compact stars at zero temperature}

For white dwarfs, the major contribution to the mass is not from the electron gas but from the non-relativistic cold nuclei: $M=2 N m_{p}$ where $m_{p}$ is the mass of a proton. There may exist other configurations that the star is almost composed of ultra-relativistic particles. In this scenario, the mass is expressed as $M=U_{0} / c^{2}$.

\subsection{White dwarfs with $M=2 N m_{p}$}

The condition $M=2 N m_{p}$ indicates that $\varepsilon_{F} \ll m_{p} c^{2}$. Therefore, $\kappa \ll 1$ for white dwarfs. The equation of state (39) shows a significant fact that the quantum gravity effects increase the degenerate pressure. This in turn induces corrections to the mass of white dwarfs. A typical model of a white dwarf has the following two properties [25]:

- The dynamics of the electrons is relativistic.

- the electron gas is completely degenerate and can be treated as a zero temperature gas.

In 3-dimensional space, we consider a star with mass $M$ and $N$ electrons. In the absence of gravitation, it is necessary to have "external walls" to keep the gas at a given density. For macro stars such as white dwarfs, it is the electronic degeneracy pressure to resist the gravitational collapse. That is, on equilibrium,

$$
P_{0}(R)=\frac{\alpha}{4 \pi} \frac{G M^{2}}{R^{4}}
$$

where $R^{3} \sim V, G$ is the constant of gravitation and $\alpha$ is a number (of the order of unity) whose exact value depends upon the distribution of matter inside stars. From eqn. (39) and eqn. (46), we have

$$
\left(\frac{N}{V}\right)^{4 / 3} h c\left(1+\delta^{2}\right)=\frac{G M^{2}}{R^{4}}
$$

where constants of the order of unity are ignored. Plugging in $M=2 N m_{p}$, the correction to the mass of stars is given by

$$
M=M_{0}\left[1+\delta^{2}\right]=M_{0}\left[1+\left(\frac{N}{V}\right)^{2 / 3} \beta h^{2}\right], \quad M_{0}=\left(\frac{1}{2 m_{p}}\right)^{2}\left(\frac{h c}{G}\right)^{\frac{3}{2}} \approx M_{\odot} .
$$


Here, $M_{0}$ is the mass of a white dwarf without corrections. For white dwarfs, $M_{0}$ is approximately equal to the Chandrasekhar limit, which is 1.44 times the mass of the sun $M_{\odot}$. One can see that the quantum gravity correction depends on the number density of the star. For a typical white dwarf, the number density of electrons is $10^{36} \mathrm{~m}^{-3}$, which gives the average distance $\bar{d}=10^{-12} \mathrm{~m}$. The Fermi energy is about $10^{5} \mathrm{eV}$ (far exceeds the white dwarf's central temperature $T \sim 10^{7} \mathrm{~K}$ ). Then (35) is well satisfied even for the upper limit of $\beta_{0} \sim 10^{36}$. Taking $\beta_{0}=10^{36}$, one finds

$$
M=M_{0}\left[1+10^{-10}\right] .
$$

Although the correction is small, one should note that the existence of quantum gravity correction is positive. Therefore, the quantum effect of gravity tends to resist the collapse of stars. Furthermore, larger $\beta_{0}$ leads to more obvious effects of quantum gravity.

\subsection{Configurations with $M=U_{0} / c^{2}$}

We consider a compact star entirely constituted by an ideal Fermi gas. The total mass is given by eqn. (29), $M=U_{0} / c^{2}$. The equilibrium equation (46) leads to

$$
R=\frac{\alpha}{24} R_{S}^{0} \tilde{h}(\kappa), \quad \tilde{h}(\kappa) \equiv \frac{\kappa^{4}}{\left(1+\kappa^{2}\right)^{2} g(\kappa)},
$$

where $R_{S}^{0}$ is the Schwarzschild radius of the star with mass $M=U_{0} / c^{2}$.

$$
R_{S}^{0}=\frac{2 G M}{c^{2}}=\frac{2 G U_{0}}{c^{4}}
$$

As shown in Figure 1, $\tilde{h}(\kappa)$ is a monotonically decreasing function of $\kappa$ with $\tilde{h}(0)=12$ and $\tilde{h}(\infty)=0$. Therefore, at a small value of $\kappa$, formation of a compact star is triggered. While at a critical value of $\kappa=\kappa^{*}$ which makes $R\left(\kappa^{*}\right)=R_{S}^{0}$, a black hole is created and thereafter. To determine $\kappa^{*}$, one has to know $\alpha$. It is conceivable that better precision can be achieved when general relativity is taken into account in the equilibrium equation (46). What is interesting is that inside the horizon, it is not a singularity but an ensemble of highly degenerate fermion gas.

It is useful to write eqn. (50) in the form:

$$
R^{2}=\frac{36 \pi}{\alpha} \beta_{0}^{2} \ell_{p}^{2} h(\kappa), \quad h(\kappa) \equiv g(\kappa) \frac{\left(1+\kappa^{2}\right)^{4}}{\kappa^{8}} .
$$

Refer to the solid line in Figure 2, the radius is divergent at $\kappa=0$ and $\kappa \rightarrow \infty$. There is a minimum $h_{\text {min }}=0.420$ around $\kappa=1.681$. Thus a minimum radius is given by

$$
R_{\min } \simeq \beta_{0} \ell_{p}=\sqrt{\beta_{0}} \Delta_{\min }
$$

\footnotetext{
${ }^{4}$ The relation between the mass and the radius of white dwarfs is calculated with the dispersion $\varepsilon=m c^{2}\left[\sqrt{1+\left(\frac{p}{m c}\right)^{2}}-\right.$ 1 ]. Since we are only concerned with the mass limit of the star configuration, the ultra-relativistic dispersion $\varepsilon=c p$ is enough. This is shown by $M_{0}$ in eqn. (48).
} 


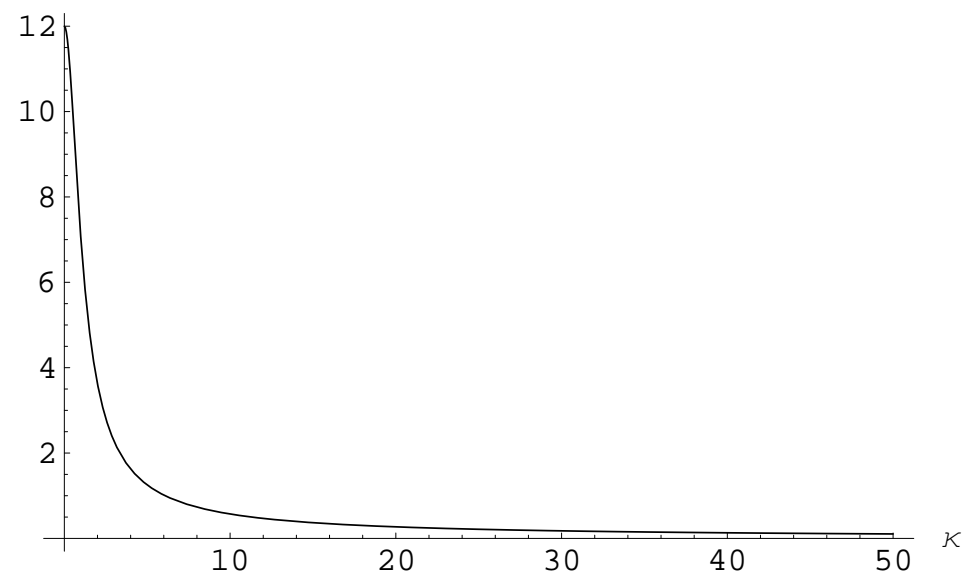

Figure 1: The $\tilde{h}(\kappa)$ in eqn. (50) versus $\kappa . \tilde{h}(\kappa)$ is a bounded and approaches zero at infinity.

which implies that no singularity is present. This conclusion is a consequence of the existence of the minimum length. Contradicting to the intuition, the minimum radius is not proportional to the minimum length but to $\sqrt{\beta_{0}} \Delta_{\text {min }}$. Nevertheless, $\beta_{0}$ may be much larger than unity as proposed by many models. Whether a star of the minimum radius is a black hole depends on the value of $\alpha$ since $\tilde{h}(1.681) \simeq 4.365$.

Substituting (52) into (25), we obtain the total number of particles, the dotted line in Figure 2 ,

$$
N=288 \sqrt{\pi}\left(\frac{1}{\alpha}\right)^{3 / 2} \beta_{0}^{3 / 2} f(\kappa) h^{3 / 2}(\kappa) .
$$

In the vicinity of $\kappa=1.290, N$ reaches a minimal value

$$
N_{\min }=N(\kappa=1.290)=0.0392 \times 288 \sqrt{\pi}\left(\frac{1}{\alpha}\right)^{3 / 2} \beta_{0}^{3 / 2} \sim \beta_{0}^{3 / 2} .
$$

Substituting (52) into (29), one acquires the total mass, the dashed line in Figure 2 ,

$$
M=\frac{U_{0}}{c^{2}}=\frac{72 \sqrt{\pi}}{\alpha^{3 / 2}} \beta_{0} M_{p} h(\kappa)^{3 / 2} \frac{\kappa^{4}}{\left(1+\kappa^{2}\right)^{2}} .
$$

Around $\kappa=0.940, M$ has a minimum

$$
M_{\text {min }}=M(\kappa=0.940)=0.107 \times \frac{2}{\sqrt{\alpha \pi}} M_{p} \beta_{0} \approx 10^{-8} \beta_{0}(\mathrm{~kg}) .
$$

The divergencies of the radius, total number of particles and mass at $\kappa=0$ are not caused by quantum gravity effects. Expanding the radius, total number of particles and mass for small $\kappa$ respectively:

$$
R=\sqrt{\frac{3 \pi}{\alpha}} \frac{(c \hbar)^{2}}{\ell_{p}} \frac{1}{\varepsilon_{F}^{2}}\left[1+\beta_{0} \frac{7}{5}\left(\frac{\varepsilon_{F}}{M_{p} c^{2}}\right)^{2}+O(\kappa)^{4}\right]
$$




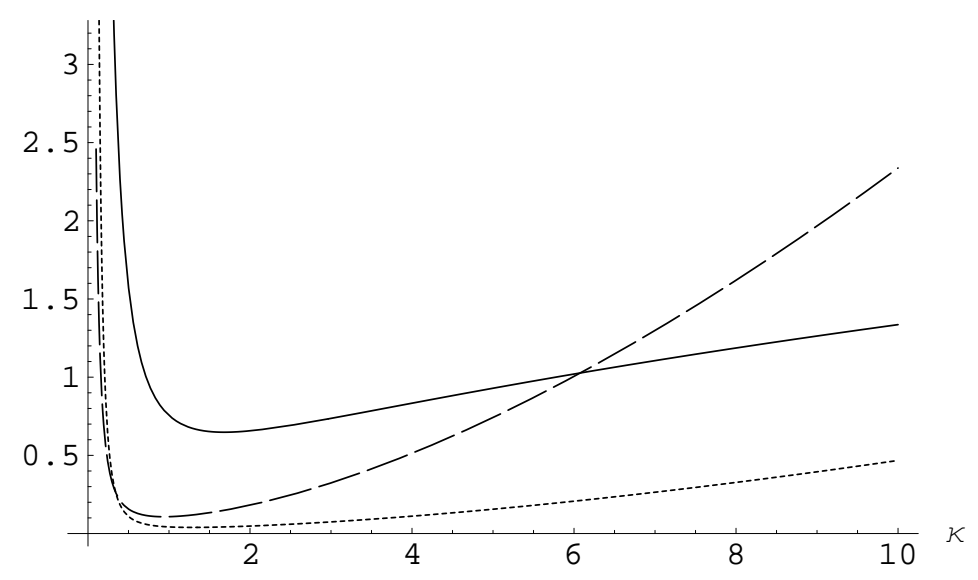

Figure 2: For a star configuration, the radius $R$ (solid line) in eqn. (52), the total number of particles $N$ (dotted line) in eqn. (54) and the mass $M$ (dashed line) in eqn. (56) versus $\kappa$. All of them are divergent at $\kappa=0$ and $\kappa \rightarrow \infty$. The radius has a minimum around $\kappa=1.681$. The total number of particles reaches a minimum around $\kappa=1.290$. The mass acquires a minimum around $\kappa=0.940$.

$$
\begin{gathered}
N=\frac{1}{4 \alpha^{3 / 2}} \sqrt{\frac{\pi}{3}}\left(\frac{c \hbar}{\ell_{p}}\right)^{3} \frac{1}{\varepsilon_{F}^{3}}\left[1+\beta_{0} \frac{12}{5}\left(\frac{\varepsilon_{F}}{M_{p} c^{2}}\right)^{2}+O(\kappa)^{4}\right] . \\
M=\frac{\sqrt{3 \pi}}{\alpha^{3 / 2}} M_{p}^{3} c^{4} \frac{1}{\varepsilon_{F}^{2}}\left[1+\beta_{0} \frac{11}{5}\left(\frac{\varepsilon_{F}}{M_{p} c^{2}}\right)^{2}+O(\kappa)^{4}\right] .
\end{gathered}
$$

The quantum gravity factor $\beta_{0}$ has no presence on the leading orders and only shows as small corrections. In fact, the ultra-relativistic dispersion $\varepsilon=c p$ is only applicable to the situation $\varepsilon_{F} \gg m c^{2}$, where $m$ is the rest mass of the particles building the star. Therefore, our results are valid in the region $\kappa \gg \sqrt{\beta_{0}} \frac{m}{M_{p}}$. When investigating the region $\varepsilon_{F} \leq m c^{2}$, the relativistic dispersion or the non-relativistic one ought to be used and one also has $\kappa \ll 1$.

As $\kappa$ keeps increasing, the Fermi pressure monotonically blows up to resist the gravitational collapse. Though both the total number of particles and mass are divergent at large $\kappa$, the number density and mass density approach finite quantities as discussed in last section. From eqn. (50) and Figure 1, beyond the critical $\kappa^{*}$, the star behaves as a black hole, in a rough definition. Eventually, the radius approaches infinity, and yet its ratio to the Schwarzschild one vanishes.

It is of interest to have a look at the asymptotic behavior of the pressure in terms of the radius. For large $\kappa$,

$$
P_{0}=\frac{\pi^{2}}{2} \frac{E_{\mathrm{H}}^{4}}{(h c)^{3}} \kappa+O(1), \quad R=\frac{3 \pi}{2 \sqrt{\alpha}} \beta_{0} \ell_{p} \sqrt{\kappa}+O\left(\frac{1}{\sqrt{\kappa}}\right)
$$

Then,

$$
\frac{d P_{0}}{d R}=\frac{\alpha}{9 \pi^{2}}\left(\frac{c}{h}\right) \frac{1}{\beta_{0}^{4} \ell_{p}^{6}} R \quad \text { as } \quad \kappa \gg 1
$$

Therefore, the increment of the Fermi pressure is accelerated for larger radius. If take the viewpoint that the whole universe is a big black hole, supported by low temperature permeating Fermi gases, the 
resulted Fermi pressure may provide a mechanism of the accelerated expansion. We anticipate that future works which make use of general relativity and interacting systems can predict observationally consistent results.

It is of importance to study the stability of the configurations. The strategy is to fix the total number of particles $N$ and vary the radius. To have a stable configuration, a positive (negative) perturbation of the radius must make the degenerate pressure smaller (larger) than the gravitational pressure. From eqns (25), (29) and (32), we have

$$
P_{0}-\frac{\alpha}{4 \pi} \frac{G M^{2}}{R^{4}}=\mathrm{const} \frac{\kappa^{8}}{\left(1+\kappa^{2}\right)^{4}} f^{-2 / 3}(\kappa)\left(j(\kappa)-j\left(\kappa_{E}\right)\right), \quad j(\kappa) \equiv h(\kappa) f^{2 / 3}(\kappa),
$$

where $\kappa_{E}$ is the value of $\kappa$ on the equilibrium for a fixed $N$. From eqn. (25), a small perturbation of the radius leads to a shift of $\kappa$ with an opposite sign:

$$
R \rightarrow R \pm|\Delta R| \Rightarrow \kappa \rightarrow \kappa \mp|\Delta \kappa| \text {. }
$$

With eqn. (61), one readily finds that in order to have stable configurations, $j(\kappa)$ must be an increasing function of $\kappa$. It is easy to see that $j(\kappa)$ has the same behavior as the dotted line, total number of particles $N$, in Fig 2, Therefore, there exists another critical value $\kappa_{S} \cong 1.290$. The star configurations are stable as $\kappa>\kappa_{S}$. The minimum radius is obviously located in the stable region. The instability of the configurations as $0 \ll \kappa \leq \kappa_{S}$ may not be very true. To have more reliable results, one should make use of the relativistic dispersion.

It is worthwhile to take a glance at a compact star comprised primarily of fermions with a small portion of bosons. This configuration is different from the two structures we have explored in this section. There does not exist any constraint between the mass and fermion number or energy. From the equilibrium condition eqn. (46), as more bosons are inhaled, the degenerate pressure $P_{0}$ and $\kappa$ move up. With eqn. (25), the volume shrinks to its minimum

$$
V_{\min }=16 \pi N \Delta_{\min }^{3},
$$

which is obviously a consequence of the existence of the minimum length. One can easily conceive that a black hole, without singularity, is going to form as the portion of bosons is growing.

\section{$5 \quad$ Summary and Discussion}

Based on the minimum observable length, we discussed the quantum gravity influences on the statistic properties of ideal gases. The finite temperature classical non-relativistic, ultra-relativistic and photon gases were addressed. Small corrections to the energy and entropy at low temperatures were found. The temperature itself is unaltered. Moreover, modifications to the equations of state may have some cosmological consequences. When connected to the traditional grand-unified string model, an lower bound $\beta_{0}>10^{4}$ was given.

We then paid attention to the ultra-relativistic Fermi gases at zero temperature. The energy density and pressure receive positive corrections proportional to $\hbar^{2} \beta(N / V)^{2 / 3}$ in the case of $\kappa \ll 1$. 
When applied to the white dwarfs, the corrections tend to resist the gravitational collapse of stars and then lift up the Chandrasekhar limit. Taking the GUP as a starting point, we also studied the high Fermi energy situation. It is shown that both the number density and the energy density approach finite values, whereas the degenerate pressure blows up for large $\kappa$.

For compact stars completely constituted by an ultra-relativistic ideal Fermi gas, we found that the radius, total number and mass all achieve their minima around $\kappa \simeq 1$. The minimum radius is the order of $\sqrt{\beta}_{0} \Delta_{\text {min. }}$. As $\kappa$ increases from a small value, a compact star is formed. After passing through a critical value $\kappa=\kappa^{*}$, where the radius coincides its Schwarzschild one, the compact star behaves as a black hole. Eventually, the radius approaches infinity, nevertheless the ratio to its Schwarzschild radius goes to zero. In this sense, the universe may be interpreted as a giant black hole. Furthermore, at large $\kappa$, the degenerate pressure increases at a rate proportional to the radius. This result provides a possible account for the accelerated expansion of the universe. The configurations are stable beyond another critical Fermi energy $\kappa_{S} \cong 1.290$.

There are several questions that we have not addressed. The first one is that in calculating the pressure balance of compact stars, we employed the Newton's gravity. At large $\kappa$, as we emphasized, it is more reasonable to include the corrections from general relativity. For instance, replace the equilibrium condition eqn. (46) by the Tolman-Oppenheimer-Volkoff (TOV) equation. Moreover, as $\kappa$ is very large, ideal gas model only serves as a leading order approximation. Better refined statistic models can certainly offer more accurate predictions. In the studies of Fermi gases and compact star configurations, we adopted zero temperature and the ultra-relativistic dispersion. It is of interest to figure out a relation between the mass and radius with relativistic dispersions . Furthermore, more

precise results are expected by assuming low but nonzero temperatures, though the calculations would be much more complicated.

We chose eqn. (6) to realize quantum gravity effects. However, whether Liouville's theorem is applicable in the high temperature limit or ultra-high density lacks of proof in principle. Atick and Witten have shown that at temperatures far above the Hagedorn temperature, string theory has a very peculiar thermodynamic behavior [23]. On the other hand, the space-time dimensionality may be reduced at short distances [26]. Therefore we naturally expect that the dimension $d$ in the equation (6) may run with energy scales. The establishment of a specific model to show the phase transition at the Hagedorn temperature will be discussed in follow-up works.

\section{Acknowledgement}

We thank Dr. X. Guo, J. Tao, B. Mu and H. Wu for the useful discussions. This work is partially supported by the Fundamental Research Funds for the Central Universities (Grant No. ZYGX2009X008), NSFC (Grant No.10705008) and NCET.

\section{References}

[1] G. Veneziano, A stringy nature needs just two constants, Europhys. Lett. 2 (1986) 199. 
[2] D. J. Gross and P. F. Mende, String theory beyond the Planck scale, Nucl. Phys. B 303 (1988) 407.

[3] D. Amati, M. Ciafaloni and G. Veneziano, Can space-time be probed below the string size? Phys. Lett. B 216 (1989) 41.

[4] K. Konishi, G. Paffuti and P. Provero, Minimum physical length and the generalized uncertainty principle in string theory, Phys. Lett. B 234 (1990) 276.

[5] R. Guida, K. Konishi and P. Provero, On the short distance behavior of string theories, Mod. Phys. Lett. A 6 (1991) 1487.

[6] M. Maggiore, A generalized uncertainty principle in quantum gravity, Phys. Lett. B 304 (1993) 65 hep-th/9301067.

[7] M. Maggiore, The algebraic structure of the generalized uncertainty principle, Phys. Lett. B 319 (1993) 83 hep-th/9309034.

[8] L. J. Garay, Quantum gravity and minimum length, Int. J. Mod. Phys. A 10 (1995) 145 [gr-qc/9403008].

[9] A. Kempf, G. Mangano and R. B. Mann, Hilbert space representation of the minimal length uncertainty relation, Phys. Rev. D 52 (1995) 1108 hep-th/9412167].

[10] A. Kempf, Quantum group-symmetric fock spaces with bargmann-fock representation, Lett. Math. Phys. 26 (1992) 1.

[11] A. Kempf, Quantum group Symmetric Bargmann-Fock space: integral kernels, Green functions, driving forces, J. Math. Phys. 34 (1994) 969.

[12] A. Kempf, Uncertainty relation in quantum mechanics with quantum group symmetry, J. Math. Phys. 35 (1994) 4483 [hep-th/9311147].

[13] S. Hossenfelder, A note on theories with a minimal length, Class. Quantum Grav. 23 (2006) 1815.

[14] S. Hossenfelder, A note on quantum field theories with a minimal length scale, Class. Quantum Grav. 25 (2008) 038003.

[15] S. K. Rama, Some consequences of the Generalised Uncertainty Principle: Statistical Mechanical, Cosmological and Varying Speed of Light, Phys. Lett. B 519 (2001) 103 hep-th/0107255.

[16] T. V. Fityo, Statistical physics in deformed spaces with minimal length, Phys. Lett. A 372 (2008) 5872 [quan-th/07120891].

[17] S. Das and E. C. Vagenas, Universality of quantum gravity corrections, Phys. Rev. Lett. 101 (2008) 221301.

[18] F. Brau and F. Buisseret, Minimal length uncertainty relation and gravitational quantum well, Phys. Rev. D 74 (2006) 036002.

[19] L. N. Chang, D. Minic, N. Okamura and T. Takeuchi, Effect of the minimal length uncertainty relation on the density of states and the cosmological constant problem, Phys. Rev. D 65 (2002) 125028 hep-th/0201017.

[20] Benrong $\mathrm{Mu}$, Houwen $\mathrm{Wu}$ and Haitang Yang, The generalized uncertainty principle in the presence of extra dimensions, arXiv:0909.3635.

[21] S. K. Rama, Dynamical features of maggiore's generalised commutation relations, hep-th/0204215

[22] K. Nozari, S. H. Mehdipour, Implications of minimal length scale on the statistical mechanics of ideal gas, Chaos, Solitons and Fractals, 32 (2007)1637-1644 hep-th/0601096. 
[23] J. J. Atick and E. Witten, The Hagedorn transition and the number of degrees of freedom of string theory, Nucl. Phys. B 310 (1988) 291.

[24] B. Zwiebach, A first course in string theory, Ref. Chap. 16, Cambridge University Press (2004).

[25] R. K. Pathria, Statistical Mechanics, Pergamon Press, First Edition, 1972.

[26] J. Ambjørn, J. Jurkiewicz and R. Loll, The Spectral Dimension of the Universe is Scale Dependent, Phys. Rev. Lett. 95 (2005) 171301. 